\title{
PAPER \\ Extended S-Parameter Method for Measuring Reflection and Mutual Coupling of Multi-Antennas
}

\author{
Takashi YANAGI $^{\dagger}{ }^{\mathrm{a})}$, Toru FUKASAWA ${ }^{\dagger}$, and Hiroaki MIYASHITA $^{\dagger}$, Members $^{\circ}$
}

\begin{abstract}
SUMMARY In this paper, a measurement method for the impedance and mutual coupling of multi-antennas that we have proposed is summarized. Impedance and mutual coupling characteristics are obtained after reducing the influence of the coaxial cables by synthesizing the measured S-parameters under the condition that unbalanced currents on the outside of the coaxial cables are canceled at feed points. We apply the proposed method to two closely positioned monopole antennas mounted on a small ground plane and demonstrate the validity and effectiveness of the proposed method by simulation and experiment. The proposed method is significantly better in terms of the accuracy of the mutual coupling data. In the presented case, the errors at the resonant frequency of the antennas are only $0.5 \mathrm{~dB}$ in amplitude and $1.8^{\circ}$ in phase.

key words: S-parameter method, multiport antennas, impedance, mutual coupling, measurement
\end{abstract}

\section{Introduction}

Traditionally, a vector network analyzer (VNA) is used to measure antenna characteristics. Coaxial cables are connected between the measurement ports of the VNA and antennas in most cases. When a coaxial cable is connected to an antenna, an unbalanced current may be induced on the outside of the cable and can cause measurement errors. These errors become larger when both the antenna and the ground plane on which the antenna is mounted are electrically small. Therefore, the cables connected to antennas require careful handling.

Ferrite chokes [1] and balun chokes [2] are well-known devices used for reducing the influence of currents on the outside of cables. However, use of these chokes presents some issues. In the former case, power absorption in ferrite chokes attached to coaxial cables causes changes in the reflection and transmission characteristics. In the latter case, wideband measurement cannot be performed at one time because the operation of balun chokes is based on the resonance characteristics of one quarter wavelength and the bandwidth is limited in principle. Additionally, in the case in which a number of antennas are closely-positioned, these chokes may not be able to be used for the measurement of mutual coupling caused by physical interference.

Another method for eliminating the influence of coaxial cables is to use fiber optics instead of metallic coaxial

Manuscript received December 22, 2015.

Manuscript revised March 31, 2016.

Manuscript publicized April 22, 2016.

${ }^{\dagger}$ The authors are with the Information Technology R\&D Center, Mitsubishi Electric Corporation, Kamakura-shi, 247-8501 Japan.

a)E-mail: Yanagi.Takashi@bp.MitsubishiElectric.co.jp DOI: $10.1587 /$ transcom.2015EBP3547 cables [3], [4]. In this case, an optical to electric (o/e) converter is required; it is mounted on the chassis on which the antennas are mounted. If the size of the chassis is smaller than the wavelength of interest, the influence of the o/e converter cannot be ignored [5].

The S-parameter method achieves frequency-independent measurements for balanced antennas [6], [7]. Two coaxial cables are connected to each element of the dipole and the full two-port S-parameters are measured. The impedance of the balanced antenna can be obtained by synthesizing these S-parameters under the condition that the unbalanced currents on the outside of the coaxial cables are canceled at the feed point. Thus, the S-parameter method has some advantages over the above-mentioned methods. First, the S-parameter method requires no additional equipment, other than two coaxial cables. Second, accurate measurements can be performed over a wide frequency range. Therefore, this method is useful for measurement of many kinds of antennas. Much research on the S-parameter method has been reported in recent years [8]-[12]. Furthermore, the S-parameter method has been applied not only to impedance measurements but also to radiation efficiency, radiation pattern, and actual gain measurements [5], [13].

The above-mentioned methods using the S-parameter method have only been applied to single-antenna measurements. However, the characteristics of multiport antennas is also important. There are many applications in which a number of antennas are mounted on a small chassis, such as mobile phones and wireless LAN cards. Recently, a decoupling and matching circuit has been used to reduce mutual coupling between closely positioned antennas [14][16]. When designing a decoupling and matching circuit, it is crucial that the amplitude and phase of reflection and the mutual coupling of antennas be measured accurately.

We have proposed an extended S-parameter method (ESPM) for the accurate measurement of reflection and mutual coupling of two or more antennas using the S-parameter method [17], [18]. In this paper, the principle and the formulation of the ESPM for reflection and mutual coupling measurement of multiport antennas are summarized and the validity of the proposed method is confirmed by both simulations and measurements. In Sect. 2, the ESPM for reflection and mutual coupling of multiport antennas is formulated. In Sect. 3, the validity of the proposed method is confirmed by simulation in the case in which two different monopole antennas are mounted on a same small chassis. In Sect. 4, measurement results for the reflection and mu- 
tual coupling characteristics of two small, strongly coupled monopole antennas are presented. The effectiveness of the proposed method is clarified by performing a comparison with the conventional method.

\section{Extended S-Parameter Method for Multiport Mea- surement}

In this section, a formulation of an ESPM applied to reflection and mutual coupling measurement for multiport antennas is described.

Consider the case in which small $n$-element antennas are mounted on a small ground plane, as shown as Fig. 1 . Two coaxial cables are connected to the antenna element and the chassis at the feed point of each antenna. Figure 2 shows the measurement model for the S-parameter method at the $k$-th antenna element $(k=1,2, \ldots, n)$. The outer conductors of each pair of coaxial cables are electrically connected near each feed point of the antennas. Here, we refer to the output port of each coaxial cable as a "coaxial cable port." The coaxial cable ports connected to the chassis and the element of ANT \# $k$ are refered to as Port: $2 k-1$ and Port: $2 k$, respectively. In this case, the $n$-element antenna system can be expressed as a $2 n$-port network. The incident waves at Port: $2 k-1$ and Port: $2 k$ are defined as $a_{2 k-1}$ and $a_{2 k}$; and the reflected waves at Port: $2 k-1$ and Port: $2 k$ are defined as $b_{2 k-1}$ and $b_{2 k}(k=1,2, \ldots, n)$, respectively. Furthermore, the port voltages are defined as $v_{2 k-1}$ and $v_{2 k}$. The port currents are defined as $i_{2 k-1}$ and $i_{2 k}$.

The incident waves, reflected waves, and S-parameters are related as follows:

$$
\mathbf{B}=\mathbf{S}^{c} \mathbf{A},
$$

where $\mathbf{S}^{c}$ is the scattering matrix of the $2 n$-port network, $\mathbf{A}$ is a column vector in which the elements are the incident waves at each port, and $\mathbf{B}$ is a column vector in which the elements are the reflected waves at each port. The superscript " $c$ " means the "coaxial cable port."

The condition under which the currents on the outside of two cables cancel each other is expressed below.

$$
i_{2 k-1}=-i_{2 k} \quad(k=1,2, \ldots, n)
$$

If the characteristic impedance is normalized to 1 , the currents and voltages can be expressed as follows:

$$
\begin{array}{ll}
i_{l}=a_{l}-b_{l} & (l=1,2, \ldots, 2 n) \\
v_{l}=a_{l}+b_{l} & (l=1,2, \ldots, 2 n) .
\end{array}
$$

From Eqs. (2) and (3), the following relationship can be obtained.

$$
a_{2 k-1}+a_{2 k}-b_{2 k-1}-b_{2 k}=0 \quad(k=1,2, \ldots, n)
$$

On the other hand, we can define the ports between the chassis and each antenna element. Here, we refer to them as "antenna port." The port voltage, port current, incident wave and reflected wave corresponding to the antenna

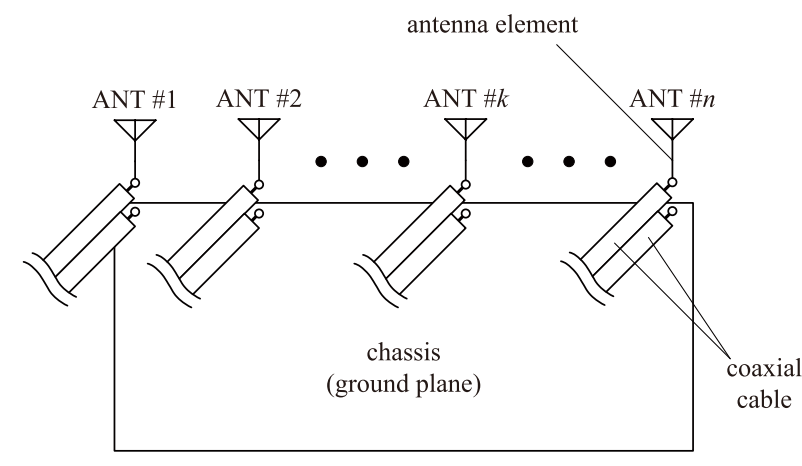

Fig. $1 n$-element antenna system.

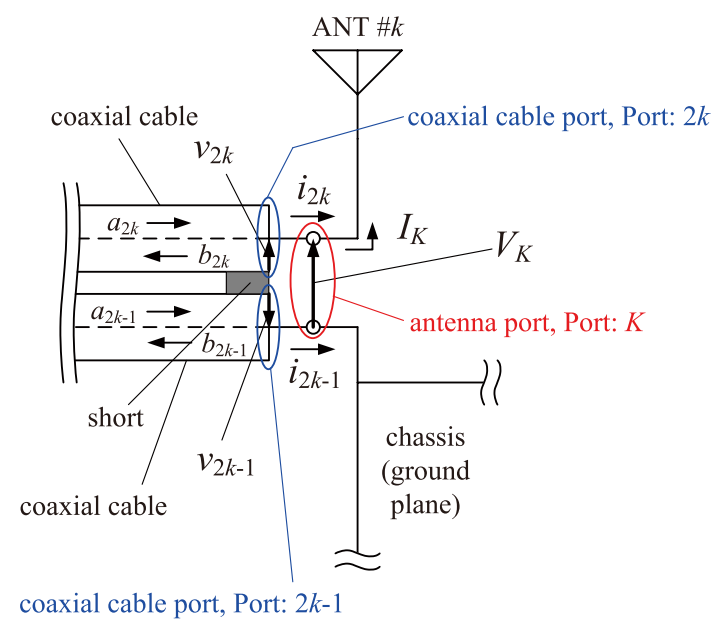

Fig. 2 Measurement model for the S-parameter method.

port are indicated by uppercase letters in order to distinguish them from the coaxial cable ports, as shown in Fig. 2. The port voltage $V_{K}$ and port current $I_{K}$ are related to $a$ and $b$ by the following equations.

$$
\begin{gathered}
V_{K}=v_{2 k}-v_{2 k-1}=\left(a_{2 k}-a_{2 k-1}\right)+\left(b_{2 k}-b_{2 k-1}\right) \\
(k=K=1,2, \ldots, n) \\
I_{K}=i_{2 k}=a_{2 k}-b_{2 k} \quad(k=K=1,2, \ldots, n)
\end{gathered}
$$

Therefore, the incident wave $A_{K}$ and the reflected wave $B_{K}$ are represented as shown below.

$$
\begin{gathered}
A_{K}=\frac{V_{K}+I_{K}}{2}=\frac{-a_{2 k-1}+2 a_{2 k}-b_{2 k-1}}{2} \\
\quad(k=K=1,2, \ldots, n) \\
B_{K}=\frac{V_{K}-I_{K}}{2}=\frac{-a_{2 k-1}-b_{2 k-1}+2 b_{2 k}}{2} \\
(k=K=1,2, \ldots, n)
\end{gathered}
$$

When $S_{1, m}^{c}, S_{2, m}^{c}, \ldots, S_{n, m}^{c}$ are to be measured, antenna ports except Port: $M$ (corresponding to ANT \# $m$ ) are set up as non-reflective terminations. Therefore, the following condition must be applied.

$$
A_{K}=0 \quad(K=1,2, \ldots, n, K \neq m)
$$


From Eqs. (8) and (10), we obtain

$$
-a_{2 k-1}+2 a_{2 k}-b_{2 k-1}=0 \quad(k=1,2, \ldots, n, k \neq m) .
$$

Thus, $4 n-1$ equations are defined on the basis of Eqs. (1), (5), and (11). Because the number of unknowns is $4 n\left(a_{1}, \ldots, a_{2 n}, b_{1}, \ldots, b_{2 n}\right)$, we can solve the system of equations by normalizing the other unknowns using one certain unknown. Here, we choose $a_{2 m-1}$, which corresponds to the incident wave at the measured port, as the unknown to normalize. The normalized unknowns are indicated by the symbol " $\hat{a}$." In this case, Eqs. (1), (5), and (11) are rewritten as follows:

$$
\begin{aligned}
& \sum_{j=1}^{2 n}\left(1-\delta_{2 m-1, j}\right) S_{i, j}^{c} \hat{a}_{j}-\hat{b}_{i}=-S_{i, 2 m-1}^{c} \\
& (i=1,2, \ldots, 2 n) \\
& \hat{a}_{2 m}-\hat{b}_{2 m-1}-\hat{b}_{2 m}=-1 \\
& \hat{a}_{2 k-1}+\hat{a}_{2 k}-\hat{b}_{2 k-1}-\hat{b}_{2 k}=0 \quad(k=1,2, \ldots, n, k \neq m) \\
& -\hat{a}_{2 k-1}+2 \hat{a}_{2 k}-\hat{b}_{2 k-1}=0 \quad(k=1,2, \ldots, n, k \neq m) \\
& \hat{a}_{i}=a_{i} / a_{2 m-1}, \hat{b}_{i}=b_{i} / a_{2 m-1} \quad(i=1,2, \ldots, 2 n)
\end{aligned}
$$

The matrix equation can be defined by combining Eqs. (12)(15), as shown below.

$$
\left[\begin{array}{cc}
\mathbf{S}^{\prime \prime} & -\mathbf{I}_{2 n} \\
\mathbf{W} & \mathbf{X} \\
\mathbf{Y} & \mathbf{Z}
\end{array}\right]\left[\begin{array}{l}
\mathbf{A}^{\prime} \\
\mathbf{B}^{\prime}
\end{array}\right]=\left[\begin{array}{c}
-\mathbf{S}_{2 m-1}^{c} \\
\mathbf{P} \\
\mathbf{O}_{n-1}
\end{array}\right]
$$

The elements of the partial matrices in Eq. (17) are described in the appendix.

The reflection coefficient at Port: $M$, namely, $S_{m m}^{a}$, and the transmission coefficient from Port: $M$ to Port: $K$, namely, $S_{k m}^{a}$, can be respectively defined using Eqs. (8) and (9) as follows:

$$
\begin{gathered}
S_{m m}^{a}=\frac{B_{m}}{A_{m}}=\frac{-1-\hat{b}_{2 m-1}+2 \hat{b}_{2 m}}{-1+2 \hat{a}_{2 m}-\hat{b}_{2 m-1}} \quad(m=1,2, \ldots, n) \\
S_{k m}^{a}=\frac{B_{k}}{A_{m}}=\frac{-\hat{a}_{2 k-1}-\hat{b}_{2 k-1}+2 \hat{b}_{2 k}}{-1+2 \hat{a}_{2 m}-\hat{b}_{2 m-1}} \\
(k=1,2, \ldots, n, m=1,2, \ldots, n, k \neq m)
\end{gathered}
$$

where the superscript " $a$ " indicates the "antenna port." Because all values of $\hat{a}$ and $\hat{b}$ can be obtained by solving the matrix equation defined by Eq. (17), the reflection coefficient and the transmission coefficient of all antennas can be calculated from Eqs. (18) and (19).

\section{Confirmation of Formulation by Simulation}

In this section, we present a simulation that was performed to confirm the validity of the formulae derived in Sect. 2. Simulated models are shown in Fig. 3(a) and (b). Two

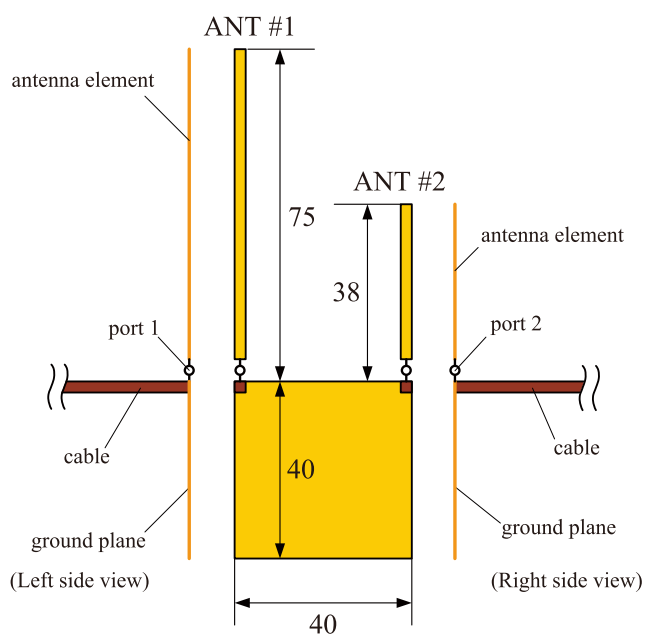

a)

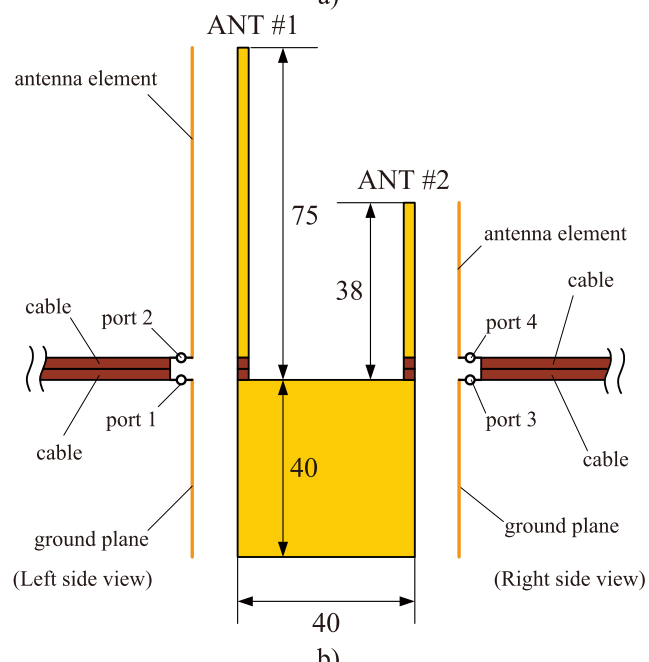

b)

Fig. 3 Simulated models for (a) conventional method and (b) $\mathrm{S}$ parameter method.

monopole antennas are mounted on each edge of a small ground plane $(40 \mathrm{~mm} \times 40 \mathrm{~mm})$. The length of ANT \# 1 is set to $75 \mathrm{~mm}$ to resonate at $950 \mathrm{MHz}$. The length of ANT \# 2 is $38 \mathrm{~mm}$ and its resonant frequency is $1.5 \mathrm{GHz}$. Conductor blocks, which correspond to coaxial cables, are included in the simulated models. In the simulation of the conventional method, the conductor blocks are connected to the ground plane near each antenna. Port 1 and port 2 are defined between the ground plane and each antenna element as shown in Fig. 3(a). On the other hand, the conductor blocks are separated from the ground plane. Ports 1 to 4 are defined as the output port of each coaxial cable as shown in Fig. 3(b). The lengths of the coaxial cables are assumed to be infinite. In this simulation, all conductors are assumed to be perfect conductors. Here, the ideal model without the coaxial cables is treated as a reference solution, and the comparison results of the conventional method and the proposed method are shown.

Figure 4 illustrates the simulation results of the reflection characteristics. The markers in Fig. 4(a) indicate the 

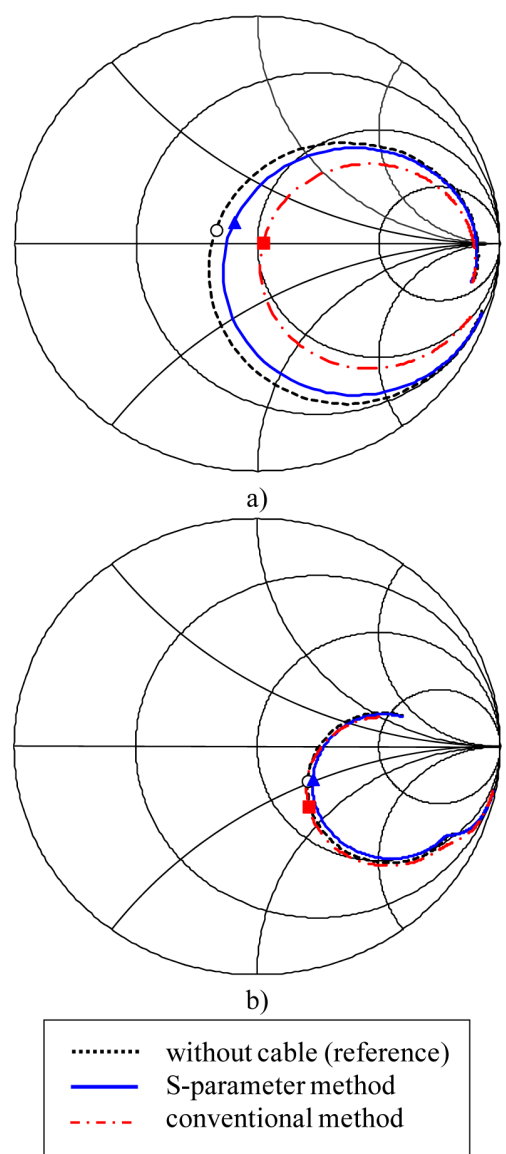

Fig. 4 Simulation results of reflection characteristics for (a) Antenna 1 and (b) Antenna 2.

resonant frequency of ANT \# 1, at $950 \mathrm{MHz}$, and those in Fig. 4(b) indicate the resonant frequency of ANT \# 2, at $1.5 \mathrm{GHz}$. In the case of ANT \# 1, the impedance locus of the conventional method is different from the reference solution. This means that the accuracy degrades because of the influence of the coaxial cables. On the other hand, it is confirmed that the accuracy is improved in the case of the proposed method. The calculated result for the proposed method is not exactly the same as the reference solution, because scattering caused by the influence of the coaxial cable remains [5].

The simulation results for the mutual coupling between ANT \# 1 and ANT \# 2 are shown in Fig. 5. In the conventional method, the error is increases with decreasing frequency. The worst amplitude error (of $3.1 \mathrm{~dB}$ ) and the worst phase error (of $11.0^{\circ}$ ) are observed. Meanwhile, both the amplitude results and the phase results are in good agreement with the reference solution. The amplitude error at $950 \mathrm{MHz}$ is $0.3 \mathrm{~dB}$.

We confirmed that the influence of the unbalanced current on the outside of the coaxial cable can be suppressed using the proposed method.

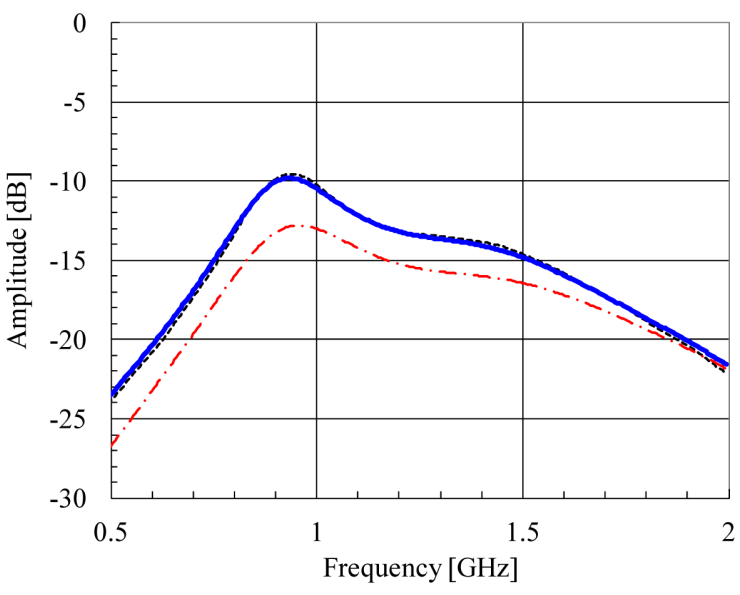

a)

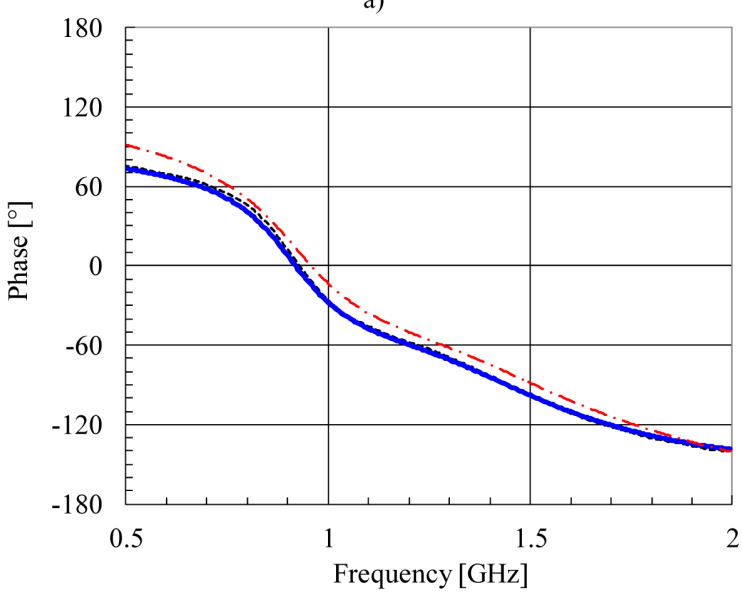

b)

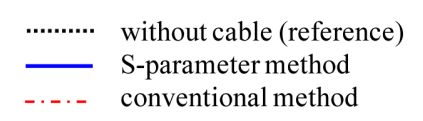

Fig. 5 Simulation results of mutual coupling between ANT \#1 and ANT \#2 for (a) amplitude and (b) phase.

\section{Measurement Results}

This section presents application of the proposed method to actual measurement for the small antennas and demonstration of its effectiveness. Figure 6 shows the configuration of the measured model. Two electrically small monopole antennas are mounted on an FR-4 substrate of dimensions $45 \mathrm{~mm} \times 40 \mathrm{~mm}$. The permittivity and loss tangent of the substrate are 4.4 and 0.02 , respectively. The ground plane is $28 \mathrm{~mm}$ in length and $40 \mathrm{~mm}$ in width. The area of each antenna is $17 \mathrm{~mm}$ (length) $\times 13 \mathrm{~mm}$ (width). The total length of each monopole is designed so as to resonate at $800 \mathrm{MHz}$; each element is bent in a spiral shape. A microstrip feed line $(50 \Omega)$ is connected to each monopole antenna. The feed ports are located at the center of the substrate.

Figure 7 shows a measurement system for the reflection coefficient and mutual coupling between two antennas. Four measurement probes made of semi-rigid cables are 


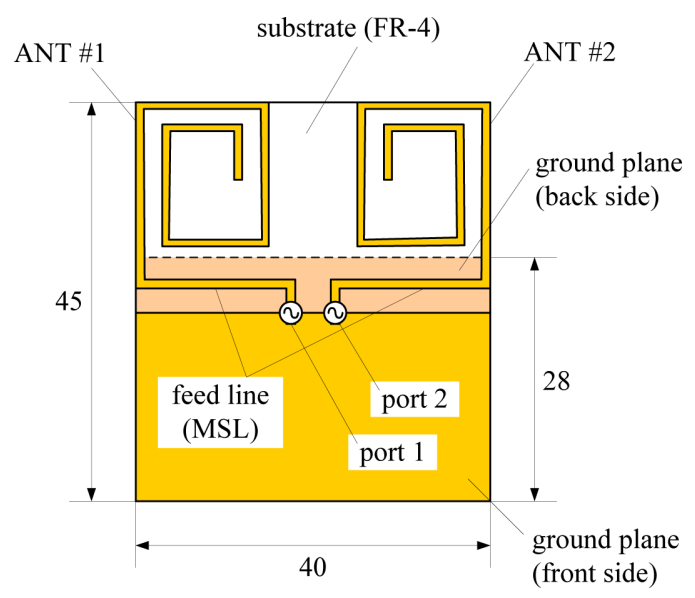

Fig. 6 Configuration of the measurement antenna model.

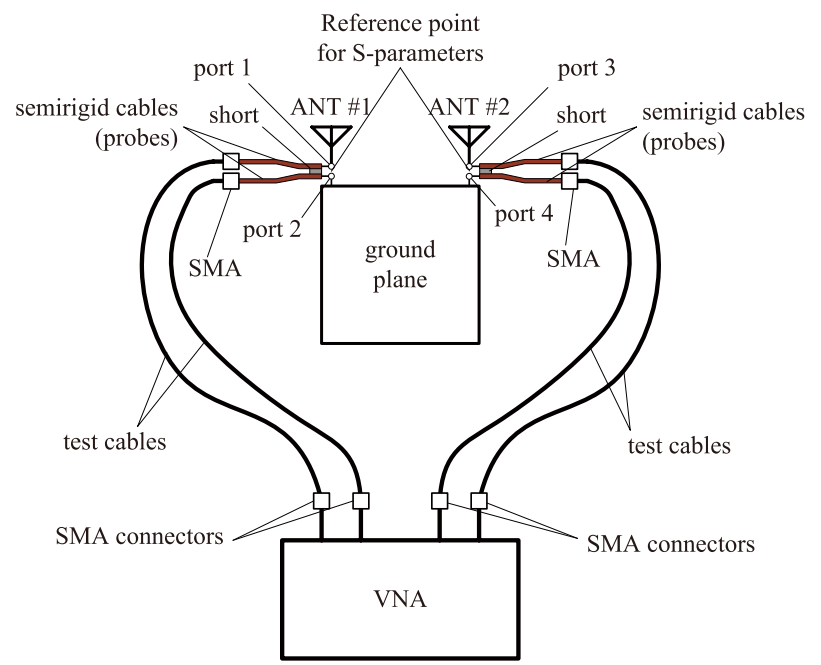

Fig. 7 Measurement system for reflection coefficient and mutual coupling between two antennas.

connected to a VNA via test cables. The other end of each probe is connected to each antenna element or the ground plane. In this case, a four-port VNA is used. Full fourport calibration is performed at the SMA connectors near the probes. Electrical delay of these measurement probes is corrected so that the reference point of S-parameters becomes at feed point of the antennas. In addition, two-port VNA can also be used. In this case, two test cables that are not connected to the VNA are terminated by a $50-\Omega$ dummy load and all S-parameters from port 1 to port 4 can be measured by changing the cables connected to the VNA.

Figure 8 shows the measurement results for the reflection coefficient and mutual coupling between two antennas. The "conventional method" refers to the case in which the coaxial cable is connected directly to each port of the antennas. The calculated reference results without coaxial cables are shown in the same figure. The markers in the Smith charts in Fig. 8 show the resonant frequencies of the antennas.

Figure $8(a)$ and (c) confirm that the resonant frequency of the antenna shifts toward a lower frequency and that an accurate measurement was not obtained by the conventional method. On the other hand, the results obtained for the proposed method are in good agreement with the calculated reference results.

When measuring mutual coupling, the accuracy can be improved by utilizing the proposed method, as shown in Fig. 8(b). The amplitude of mutual coupling at $800 \mathrm{MHz}$ is $3 \mathrm{~dB}$ lower than the calculated reference result for the conventional method. Meanwhile, the error is only $0.5 \mathrm{~dB}$ in the case of using the proposed method. Furthermore, the phase characteristics obtained using the proposed method agree with the calculated reference result over the whole range of measured frequencies, whereas a difference of approximately $23^{\circ}$ was observed in the case of using the conventional method.

\section{Conclusion}

We have proposed an accurate measurement method (by applying S-parameter method) for the mutual coupling between antennas mounted to the same small ground plane. Two coaxial cables are connected to an antenna element and the ground plane of each antenna. The impedance characteristics and mutual coupling with reduced unbalanced currents can be obtained by synthesizing the S-parameters under the condition that the unbalanced currents on the outside of the coaxial cables are canceled at the feed points.

We have analyzed a simulated model that includes two asymmetric monopole antennas with cables and a small ground plane and have compared the proposed method to the conventional method. The frequency characteristics of mutual coupling between the two antennas obtained using the proposed method agree closely with the simulation results in the ideal case, namely where no cables exist.

Moreover, we have applied the proposed method to real-world measurements and have demonstrated that the measurement accuracy can be improved (relative to the conventional method). In particular, the proposed method is superior in terms of the accuracy of the mutual coupling data. In this case, the errors at the resonant frequency of the antennas are only $0.5 \mathrm{~dB}$ in amplitude and $1.8^{\circ}$ in phase. This method is especially effective for designing decoupling and matching circuits for small antennas.

\section{References}

[1] S. Saario, D.V. Thiel, J.W. Lu, and S.G. O'Keefe, “An assessment of cable radiation effects on mobile communications antenna measurements," Proc. IEEE Antennas and Propagation Society International Symposium 1997, pp.550-553, 1997.

[2] C. Icheln, J. Krogerus, and P. Vainikainen, "Use of Balun chokes in small-antenna radiation measurements," IEEE Trans. Instrum. Meas., vol.53, no.2, pp.498-506, April 2004.

[3] T. Fukasawa, K. Shimomura, and M. Ohtsuka, "Accurate measurement method using fiber-optics for an antenna on a portable telephone," IEEE Topical Conference on Wireless Communication Technology, pp.138-139, 2003. 

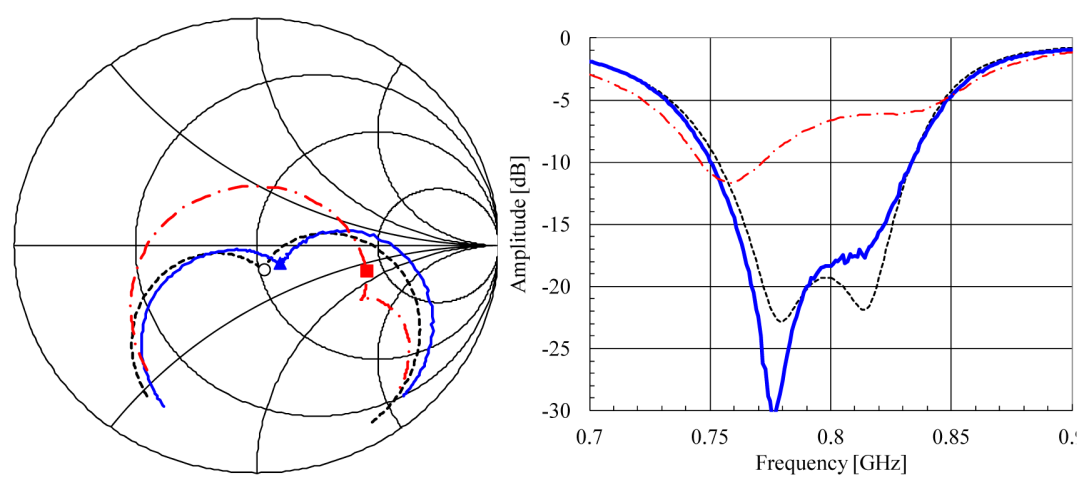

a)
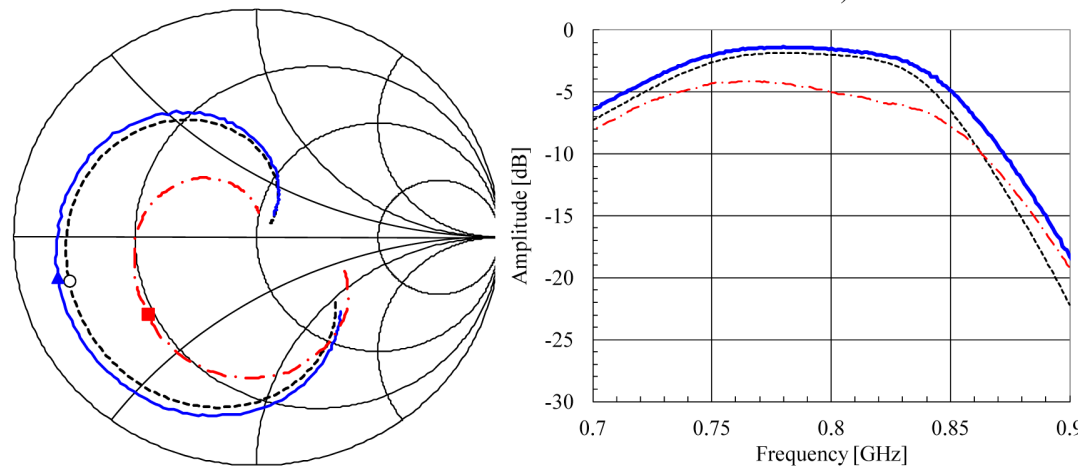

b)
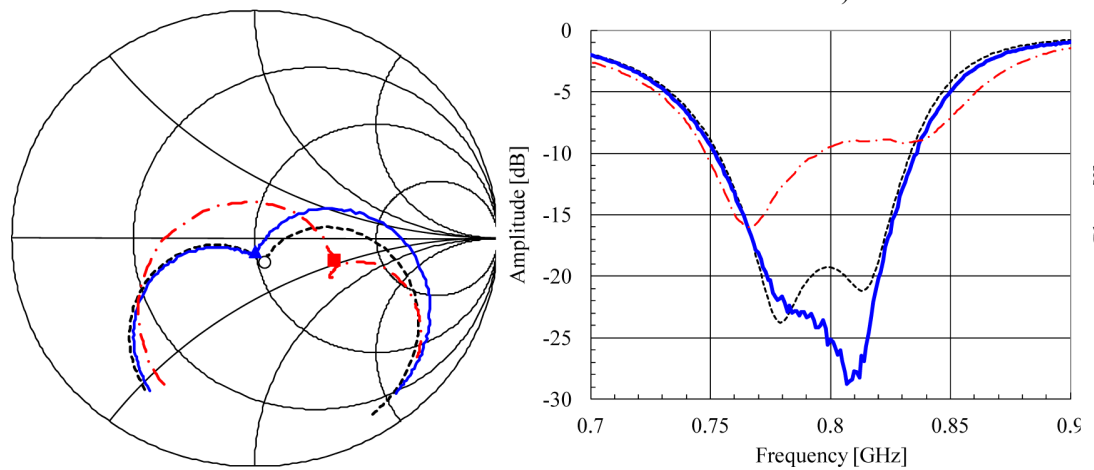

c)

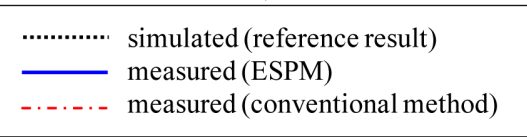

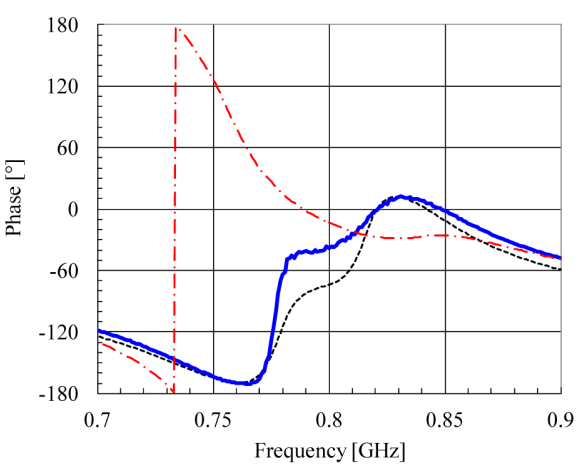
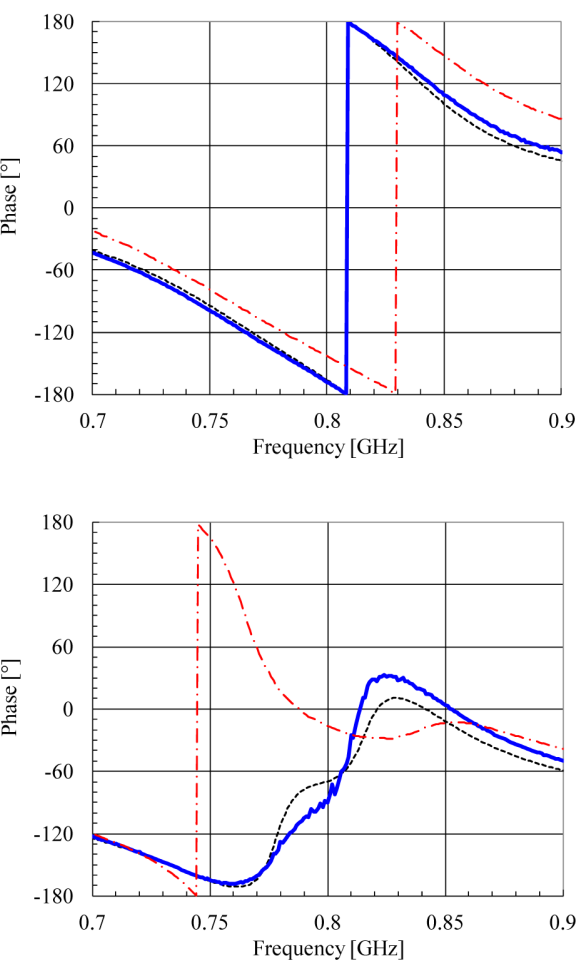

Fig. 8 Measurement results for reflection characteristics and mutual coupling for (a) S11, (b) S21, and (c) S22. The left, center, and right figures show the Smith chart, and the frequency characteristics for amplitude and phase, respectively.

[4] T. Fukasawa, K. Shimomura, and M. Ohtsuka, "Accurate measurement method for characteristics of an antenna on a portable telephone," IEICE Trans. Commun. (Japanese Edition), vol.J86-B, no.9, pp.1895-1905, Sept. 2003.

[5] T. Fukasawa, T. Yanagi, H. Miyashita, and Y. Konishi, "Extended S-parameter method including radiation pattern measurements of an antenna," IEEE Trans. Antennas Propag., vol.60, no.12, pp.56455653, Dec. 2012.

[6] R. Meys and F. Janssens, "Measuring the impedance of balanced antennas by an S-parameter method," IEEE Antennas Propag. Mag., vol.40, no.6, pp.62-65, Dec. 1998.

[7] K.D. Palmer and M.W. van Rooyen, "Simple broadband measurements of balanced loads using a network analyzer," IEEE Trans. Instrum. Meas., vol.55, no.1, pp.266-272, Feb. 2006.
[8] M. Sun, Y.P. Zhang, K.M. Chua, L.L. Wai, D. Liu, and B.P. Gaucher, "Integration of Yagi antenna in LTCC package for differential 60GHz radio," IEEE Trans. Antennas Propag., vol.56, no.8, pp.27802783, Aug. 2008

[9] X. Qing, C.K. Goh, and Z.N. Chen, "Impedance characterization of RFID tag antennas and application in tag co-design," IEEE Trans. Microw. Theory Techn., vol.57, no.5, pp.1268-1274, May 2009.

[10] A.G. Alhaddad, R.A. Abd-Alhameed, D. Zhou, C.H. See, I.T.E Elfergani, and P.S. Excell, "Low profile dual-band-balanced handset antenna with dual-arm structure for WLAN application," IET Microw. Antennas Propag., vol.5, no.9, pp.1045-1053, June 2011.

[11] J. Sarrazin, Y. Mahe, S. Avrillon, and S. Toutain, "A new multimode antenna for MIMO systems using a mode frequency convergence concept," IEEE Trans. Antennas Propag., vol.59, no.12, pp.4481- 
4489, Dec. 2011.

[12] M. Pigeon, R. D'Errico, and C. Delaveaud, "UHF-UWB tag antenna for passive RFID applications," EuCAP 2013, pp.3968-3972, April 2013.

[13] J. Zhang, S. Pivnenko, and O. Breinbjerg, "A modified wheeler cap method for radiation efficiency measurement of balanced electrically small antennas," EuCAP 2010, pp.12-16, April 2010.

[14] S. Dossche, S. Blanch, and J. Romeu, "Optimum antenna matching to minimise signal correlation on a two-port antenna diversity system," Electron. Lett., vol.40, no.19, pp.1164-1165, Sept. 2004.

[15] S.-C. Chen, Y.-S. Wang, and S.-J. Chung, "A decoupling technique for increasing the port isolation between two strongly coupled antennas," IEEE Trans. Antennas Propag., vol.56, no.12, pp.3650-3658, Dec. 2008

[16] C. Luxey, "Design of multi-antenna systems for UMTS mobile phones," Proc. 2009 Loughborough Antennas \& Propagation Conference, pp.57-64, 2009.

[17] T. Yanagi, T. Fukasawa, H. Miyashita, and Y. Konishi, "Measurement using the S-parameter method for radiation characteristics and mutual coupling of multiport antennas on a small ground," Proc. 2012 Int. Symp. Antennas and Propagation, pp.1003-1006, Nov. 2012.

[18] T. Yanagi, T. Fukasawa, and H. Miyashita, "Extended S-parameter method for radiation characteristics and mutual coupling of multiport antennas," Proc. 2013 Progress in Electromagnetics Research Symposium, pp.829-832, Aug. 2013.

\section{Appendix: Partial Matrices of Eq. (17)}

The partial matrices of Eq. (17) are described below. $\mathbf{S}^{c \prime}$ is a matrix defined by removing the $(2 m-1)$-th column from Eq. (1):

$$
\begin{aligned}
S_{i, j}^{c^{\prime}}= \begin{cases}S_{i, j}^{c} & (j<2 m-1) \\
S_{i, j+1}^{c} & (j \geq 2 m-1)\end{cases} & (i=1,2, \ldots, 2 n, j=1,2, \ldots, 2 n-1)
\end{aligned}
$$

$\mathbf{A}^{\prime}$ is a column vector in which the elements represent the normalized incident wave:

$$
A_{i}^{\prime}=\left\{\begin{array}{ll}
\hat{a}_{i} & (i<2 m-1) \\
\hat{a}_{i+1} & (i \geq 2 m-1)
\end{array} \quad(i=1,2, \ldots, 2 n-1)\right.
$$

$\mathbf{B}^{\prime}$ is a column vector in which the elements represent the normalized reflected wave:

$$
B_{i}^{\prime}=\hat{b}_{i} \quad(i=1,2, \ldots, 2 n)
$$

$\mathbf{S}_{2 m-1}^{c}$ is a column vector that consists of the $(2 m-1)$-th column of the scattering matrix:

$$
\left(S_{2 m-1}^{c}\right)_{i}=S_{i, 2 m-1}^{c} \quad(i=1,2, \ldots, 2 n)
$$

$\mathbf{W}, \mathbf{X}$, and $\mathbf{P}$ are matrices or a column vector defined by Eqs. (13) and (14):

$$
W_{i, j}= \begin{cases}1 & \left.\begin{array}{cc}
j=2 i-1,2 i & (i<m) \\
j=2 i-1 & (i=m) \\
j=2 i-2,2 i-1 & (i>m)
\end{array}\right) \\
0 \quad \text { (else) } & \end{cases}
$$

$$
\begin{gathered}
(i=1,2, \ldots, n, j=1,2, \ldots, n-1) \\
X_{i, j}= \begin{cases}-1 & (j=2 i-1,2 i) \\
0 & (\mathrm{else})\end{cases} \\
P_{i}=\left\{\begin{array}{ll}
-1 & (i=1,2, \ldots, n, j=1,2, \ldots, n) \\
0 & (\mathrm{else})
\end{array} \quad(i=1,2, \ldots, n)\right.
\end{gathered}
$$

$\mathbf{Y}$ and $\mathbf{Z}$ are matrices defined by Eq. (15):

$$
\begin{aligned}
& Y_{i, j}=\left\{\begin{array}{lll}
-1 & \left(\begin{array}{cc}
j=2 i-1 & (i<m) \\
j=2 i & (i \geq m)
\end{array}\right) \\
2 & \left(\begin{array}{cc}
j=2 i & (i<m) \\
j=2 i+1 & (i \geq m)
\end{array}\right) \\
0 & (\text { else }) &
\end{array}\right. \\
& (i=1,2, \ldots, n-1, j=1,2, \ldots, 2 n) \\
& Z_{i, j}=\left\{\begin{array}{lll}
-1 & \left(\begin{array}{ll}
j=2 i-1 & (i<m) \\
j=2 i+1 & (i \geq m)
\end{array}\right) \\
0 & \text { (else) }
\end{array}\right. \\
& (i=1,2, \ldots, n-1, j=1,2, \ldots, 2 n)
\end{aligned}
$$

Finally, $\mathbf{I}_{2 n}$ is an identity matrix of $2 n$ order and $\mathbf{O}_{n-1}$ is a zero vector of $(n-1)$ order.

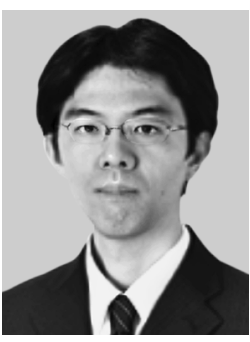

Takashi Yanagi was born in Tokyo, Japan on January 21, 1979. He received B.S. and M.S. degrees in electrical engineering from Waseda University, Tokyo, Japan, in 2002 and 2004, respectively. He joined Mitsubishi Electric Corporation, Tokyo, Japan, in 2004. Since then, he has been engaged in the research and development of antennas for mobile communication. He is currently with the Antenna Technology Department of the Information Technology R\&D Center, Mitsubishi Electric Corporation. He received the Young Researcher's Award from the IEICE in 2011 and the 2012 Best Paper Award from the IEICE. He is a member of the IEEE.

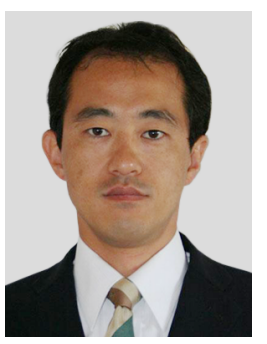

Toru Fukasawa was born in Tokyo, Japan, on Dec. 24, 1969. He received B.S., M.S., and Ph.D. degrees in electronic engineering from Hokkaido University, Sapporo, Japan, in 1992, 1994, and 2003, respectively. He joined Mitsubishi Electric Corporation, Tokyo, Japan, in 1994. His primary research activities are in small antenna analysis and measurement methods. Currently, he is the Manager of the Antennas Technology Department, Information Technology R\&D Center, Mitsubishi Electric Corporation. He received the Young Researcher's Award from The IEICE in 1999 and the 2011 and 2012 Best Paper Awards from the IEICE. He is a member of the IEEE. 


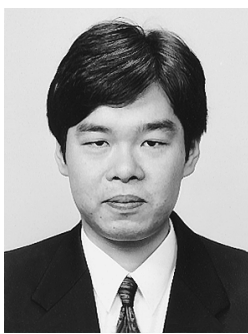

Hiroaki Miyashita was born in Shimoda, Shizuoka, Japan, on May 2, 1964. He received the B.S. degree in applied physics from the University of Tokyo, Japan, in 1987 and the Ph.D. degree from Kyoto University, Kyoto, Japan, in 2000. He joined Mitsubishi Electric Corporation, Tokyo, Japan, in 1988. His primary research activities are in antenna analysis and applied mathematics for wave phenomena. Presently, he is the Senior Manager of the Antennas Technology Department, Information Technology R\&D Center, Mitsubishi Electric Corporation. Dr. Miyashita received the IEEE Antennas and Propagation Society Tokyo Chapter Young Engineer Award in 1994, the Young Researcher's Award from the IEICE in 1995, and the 2011 and 2012 Best Paper Awards from the IEICE. He is a senior member of the IEEE, the American Physical Society, and the American Mathematical Society. 